\title{
A Comprehensive Development Agenda on Tenofovir Alafenamide in Clinical Practice
}

\begin{abstract}
Antonio Di Biagio ${ }^{1}$, Niccolò Riccardi' ${ }^{1}$, Stefano Rusconi², Giovanni Guaraldi ${ }^{3}$, Marco Borderi ${ }^{4}$, Andrea De Luca ${ }^{5}$, Nicola Gianotti ${ }^{6}$, Sergio Lo Caputo7, Paolo Maggi ${ }^{7}$, Renato Maserati ${ }^{8}$ and Franco Maggiolo ${ }^{9}$

${ }^{1}$ Clinic of Infectious Diseases, Ospedale Policlinico San Martino, Genova; ${ }^{2}$ Divisione Clinicizzata di Malattie Infettive, DIBIC Luigi Sacco, University of Milan, Milan; ${ }^{3}$ Associate Professor of Infectious Diseases, University of Modena and Reggio Emilia; ${ }^{4}$ Infectious Disease Unit, Department of Medical and Surgical Sciences, Alma Mater Studiorum, University of Bologna, Bologna; ${ }^{5}$ Division of Infectious Diseases, Siena University Hospital, Siena; ${ }^{6}$ Dipartimento di Malattie Infettive, San Raffaele Scientific Institute, Milan; ${ }^{7}$ Clinic of Infectious Diseases, Università Degli Studi di Bari Aldo Moro, Bari; ${ }^{8}$ Department of Infectious Diseases, Foundation I.R.C.C.S “San Matteo Hospital", Pavia; ${ }^{9}$ Division of Infectious Diseases, ASST Papa Giovanni XXIII, Bergamo. Italy
\end{abstract}

\begin{abstract}
The introduction of tenofovir (TFV) alafenamide (TAF) into clinical practice will be a further revolution in antiretroviral therapy. Currently available HIV-1 regimens are wide enough to allow diversified usage in different settings. Despite the fact that TAF is not capillary accessible, even in industrialized countries, ultimate International Guidelines have already included TAF in backbone or in single-tablet regimens. Due to a better safety profile, TAF will progressively replace TFV disoproxil fumarate, both in naïve and experienced patients. However, therapeutic innovations have to deal with budget constraints and different global spending-review patterns. The aim of this article is to give a comprehensive agenda of TAF use in naïve and experienced HIV-1 infected patients, providing a full review of the studies present in the literature and contextualizing these findings into daily clinical practice. (AIDS Rev. 2018;20:75-82) Corresponding author: Antonio Di Biagio, adibiagioa@gmail.com/antonio.dibiagio@hsanmartino.it
\end{abstract}

\section{Key words}

Tenofovir alafenamide. Tenofovir disoproxil fumarate. Combination antiretroviral treatment. Antiretroviral strategies.

\section{Introduction}

The combination antiretroviral treatment (cART) regimens containing tenofovir (TFV) alafenamide (TAF), the novel pro-drug of the nucleotide reverse transcrip-

Correspondence to:

Antonio Di Biagio,

Clinica Malattie Infettive,

Ospedale Policlinico San Martino,

Padiglione Specialità Complesse,

Piano -2, Largo R. Benzi 10, 16132 Genova

E-mail: adibiagioa@gmail.com tase inhibitor (NRTI) TFV, are recommended for the treatment of naïve and experienced patients by national and international guidelines ${ }^{1,2}$.

Since its introduction, TAF has demonstrated high efficacy and tolerability in the treatment of people living
Received in original form: $28 / 08 / 2017$

Accepted in final form: 19/02/2018

DOI: 10.24875/AIDSRev.M18000017 
with HIV (PLWHIV) when used in combination with other antiretroviral agents ${ }^{3-5}$. TAF, due to lower circulating levels of TFV, but higher concentrations of its metabolically active form TFV-DP, inside lymphoid cells, ensures a better safety profile compared to TFV disoproxil fumarate (TDF) and may play a key role in reducing bone and renal CART-related toxicity in PLWHIV6-8.

In Europe, in a short-time have been launched on the market two different single-tablet regimen (STR) containing TAF (elvitegravir [EVG]/cobicistat [C]/emtricitabine [FTC] and rilpivirine [RLP]/FTC) and a fixed dose containing FTC/TAF, and other TAF-based combinations will be launched in the near future (Table 1).

In antiretroviral naïve patients and antiretroviral experienced patients already in TDF-based regimen, TAF will replace TDF progressively when patients are linked to care. There are more than 35 million PLWHIV and 15 million are receiving $\mathrm{CART}^{9}$; the majority of them is on treatment with TDF in combination with FTC or lamivudine ${ }^{10}$, therefore, to avoid excessive costs, to minimize long-term cART related side effects and to guarantee a further treatment option in all patients, it should be suggested to prioritize switching strategies from TDF to TAF. In the near future, even experienced patients currently treated with TDF-free regimen may benefit of switching to TAF.

The aim of this review is to give a comprehensive agenda of TAF use in naivve and experienced HIV-1 infected patients.

\section{What is TAF?}

TAF, originally named GS-7340, is a novel NRTI, prodrug of TFV, with a more stable blood and plasma pharmacokinetic profile that allows targeted intracellular accumulation of TDF and consequent phosphory- lation to the active metabolite TFV-DP Figure $1^{6}$. Due to higher intracellular levels of TFV-DP and lower circulating levels of TFV compared to TDF, TAF has shown a more favorable pharmacological profile and a higher antiviral activity at lower doses than its parent drug ${ }^{11}$. Moreover, due to lower circulating levels of TFV, TAF minimizes the short- and long-term impact on estimated creatinine clearance, renal tubular proteinuria, and bone mineral density (BMD), thus, certifying its superiority compared to TDF ${ }^{12}$.

In vitro studies have demonstrated a higher inhibitory quotient of TAF than TDF, the implication of this on clinical practice may result in the potential of TAF to inhibit HIV-1 viruses resistant to TDF ${ }^{13}$.

\section{Initial cART with TAF-based regimen in naïve patients}

TAF-based options for initial CART in naîve adults patients include an STR with E/C/FTC/TAF, or dolutegravir (DTG) plus the backbone with FTC/TAF or raltegravir (RAL) plus FTC/TAF or in patients with HIV-RNA load $<100,000$ copies/mL, and CD4+ cell count $>200$ cells/ $\mathrm{mmc}$ a rilpivirine based STR (RPV/FTC/TAF) ${ }^{2-14}$

E/c/FTC/TAF, named Genvoya ${ }^{\circledR}$ by Gilead Sciences, was approved by FDA and by EMA in November 2015 in naïve adults and adolescent patients (12 years and older and of at least $35 \mathrm{Kg}$ body weight $)^{15,16}$, in this STR the TAF dose is $10 \mathrm{mg}$. RPV/F/TAF, named Odefsey ${ }^{\circledR}$ by Gilead Sciences, was approved by EDA in March 2016, and by EMA in April 2016, for the use in adults and adolescents (aged 12 years and older with body weight at least $35 \mathrm{~kg}$ ) without known mutations associated with resistance to the non-nucleoside reverse transcriptase inhibitor class (NNRTI), TDFor FTC and with a VL $\leq 100,000$ copies/mL. ${ }^{17,18}$ RPV/FTC/TAF

\begin{tabular}{|c|c|c|c|c|}
\hline Drug & EMA approved & Therapeutic area & Study patients & $\stackrel{\circ}{\cdots}$ \\
\hline EVG/c/FTC/TAF & November 2015 & HIV & Naïve and experienced & 을 \\
\hline FTC/TAF (25 mg or $10 \mathrm{mg}$ ) & April 2016 & HIV & Switch studies & $\cong$ \\
\hline RPV/FTC/TAF & June 2016 & HIV & Bioequivalence & 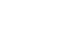 \\
\hline TAF & January 2017 & HBV & Naïve and experienced & $\stackrel{\sqrt{2}}{2}$ \\
\hline Darunavir/c/FTC/TAF & na & HIV & Naive & 7 \\
\hline Bictegravir/FTC/TAF & na & HIV & Naive & \\
\hline
\end{tabular}




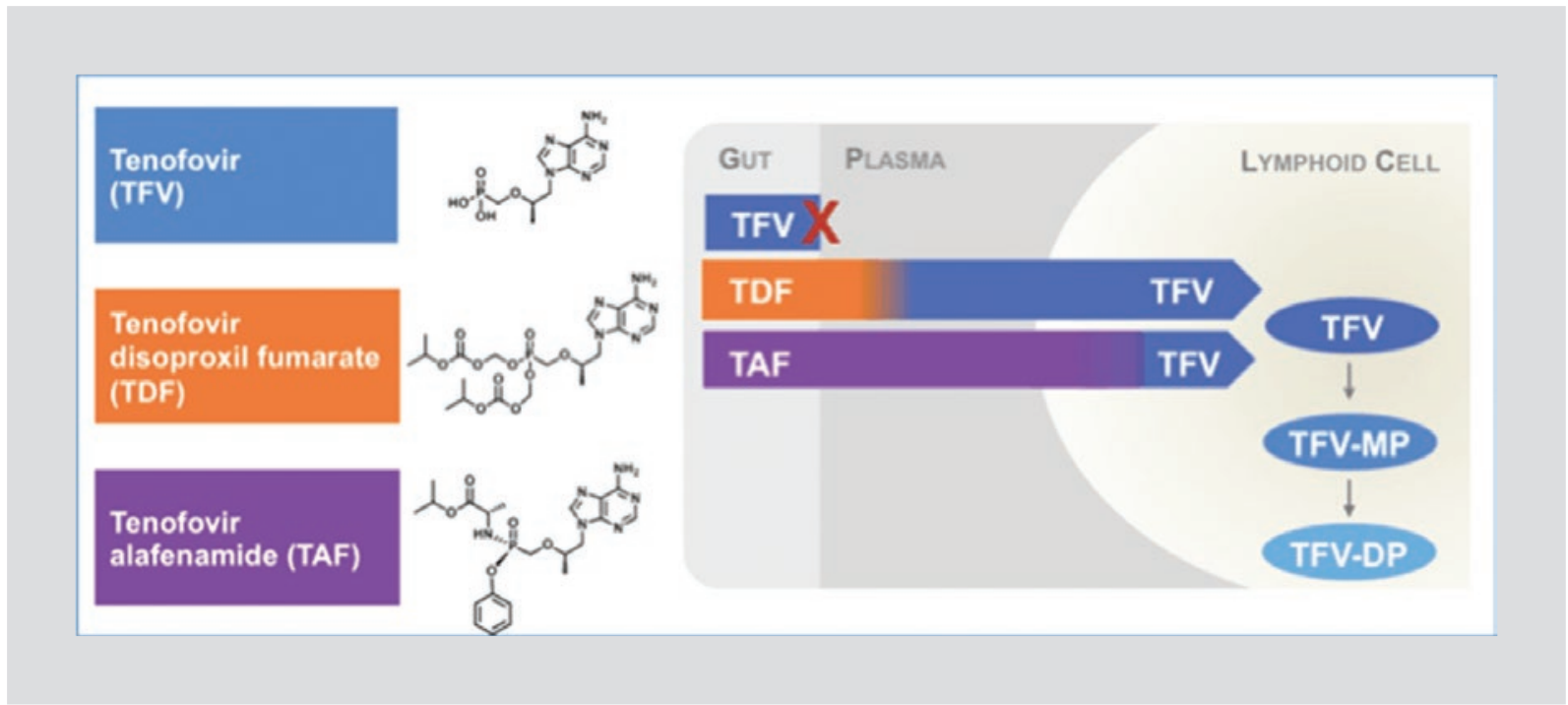

Figure 1. Mechanism of action for tenofovir disoproxil fumarate and tenofovir alafenamide.

contains $25 \mathrm{mg}$ of TAF. The new backbone combination FTC/TAF, named Descovy ${ }^{\circledR}$ by Gilead Sciences, was approved by FDA and by EMA in April 2016 in combination with other antiretroviral agents in adults and adolescents patients (12 years of age and older) ${ }^{3,19}$. Descovy may be used either with $25 \mathrm{mg}$ (when an unboosted third agent is associated) or 10 mg of TAF. In the next future, a new STR containing darunavir $800 \mathrm{mg} / \mathrm{c} / \mathrm{FTC} / \mathrm{TAF}$, by Janssen-Cilag International NV, will be available ${ }^{20}$. This new fixed-dose combination contains $10 \mathrm{mg}$ of TAF.

\section{Why a TAF-based regimen in naïve patients?}

Efficacy and safety of TAF compared to TDF have been established, creating the necessary conditions for the transition to the new backbone with FTC/TAF ${ }^{21,22}$.

The first two clinical efficacy trials that compared TAF with TDF, in naïve patients, were two controlled, doubleblind phase 3 noninferiority studies, registered on ClinicalTrials.gov, numbers NCT01780506 and NCT0179744523.1,733 treatment naïve patients with an HIV-RNA > 1,000 copies/mL, no resistance to E, FTC and TDF in GRT and estimated glomerular filtration of $50 \mathrm{~mL} / \mathrm{min}$ or higher from 178 outpatient centers in 16 countries were randomly assigned E/C/FTC/TAF (866 patients) or E/C/FTC/TDF (867 patients). The primary outcomes were the percentage of patients with HIV-RNA $<50$ copies $/ \mathrm{mL}$ at week 48 , as defined by the FDA snapshot analysis (noninferiority margin of $12 \%$ ) and renal and bone safety endpoints at week 48. After
48 weeks of treatment, efficacy profiles were comparable with a HIV-RNA < 50 copies/mL in more than $90 \%$ of patients in the E/C/F/TAF arm (differences 2.0\%, 95\% confidence interval $[\mathrm{Cl}]-0.7-4.7 \%)$. E/C/F/TAF noninferiority was also observed in relation to HIV-RNA $<20$ copies/mL with very high 96 weeks virologic suppression rates (84.4\%: differences $0.4 \%, 95 \% \mathrm{Cl}:-3.0-3.8 \%$ and $81.5 \%$ : differences $1.5 \%, 95 \% \mathrm{Cl}-2.2-5.2 \%$ ), respectively. At week 48 , the mean CD4 count increase was higher in the TAF based regimen arm: $230 \mathrm{cell} / \mathrm{mmc}$ versus $211 \mathrm{cell} / \mathrm{mmc}(p=0.024)$. Virological failure occurred in $4 \%$ of HIV-infected patients in each treatment arm, and resistance in 7 patients $(0.8 \%)$ in the TAFbased regimen arm versus $5(0.6 \%)$ in the TDFbased arm. At week 96 , the results were confirmed $(86.6 \%$ in TAF arm vs. $85.2 \%$ in TDF based arm with HIV-RNA < 50 copies $/ \mathrm{mL}$; difference $1.5 \%, 95 \% \mathrm{Cl}-1.8-4.8 \%$ ). The adverse event rates through 96 weeks were similar in two groups, and most events reported were mild or moderate. However, renal side effects were decreased in the E/C/F/TAF arm with a median increase of serum creatinine of 0.08 for the TAF arm compared to $0.12 \mathrm{mg} /$ $\mathrm{dL}$ in the TDF arm $(p<0.0001)$ and a median $\%$ change in proteinuria of -3 in the TAF arm compared to 20 in the TDF arm $(p<0.0001)$. In the TAF arm, a significant smaller decrease in BMD at spine and hip was noticed after 48 weeks of therapy ${ }^{23}$. Furthermore, no cases of proximal tubulopathy for TAF were noticed, while four cases in the TDF arm. The safety and efficacy were preserved after 144 weeks of therapy ${ }^{24}$.

Similarly, fixed-dose STR containing TAF/FTC has been studied in a randomized, double-blinded multi- 
center active-controlled study (ClinicalTrials.gov, number NCT01565850). 232 treatment-naive adults were screened, 153 were randomized 2:1 to Darunavir/c/F/ $\operatorname{TAF}(n=103)$ versus Darunavir/c + F/TDF $(n=50)$. At both weeks 24 and 48, there were no significant differences in virologic suppression: $76.7 \%$ on TAF versus $84.0 \%$ on TDF by pre-specified subgroup analyses by demographic and disease characteristics, or adherence.

The AMBER study registered at clinicaltrials.com as NCT02431247 is evaluating noninferiority in efficacy and safety of darunavir/c/FTC/TAF fixed-dose combination versus darunavir/c/FTC/TDF in naïve patients ${ }^{25}$.

The TAF arm had significantly improved renal and bone safety parameters: less proteinuria and less change in hip and spine BMD, consistent with results from a similarly designed study of the E/C/F/TAF 26,27 . Moreover, long-term safety, durability as well as tolerability make TAF/FTC based regimen a favorable and desirable choice for initial CART with a low risk of developing drug resistance ${ }^{14-30}$. To date, studies are ongoing in naive patients evaluating the efficacy and safety of TAF in fixed-dose combination with FTC and a novel INSTI, named bictegravir (GS-9883), compared to TAF/FTC plus DTG ${ }^{31}$.

This recommendation follows the fact that TAF/FTC, as just recalled, has shown non-inferior efficacy as compared to TDF/FTC in combination with EVG/cobicistat and, in a smaller study, with darunavir/cobicistat. Given these results and the reduced renal and bone toxicity, this combination should be preferred to TDF/ FTC for all first-line regimens, particularly, those with a boosted protease inhibitor or integrase inhibitor as third agent. Compared with the other nucleoside fixed-dose combination available on the market (i.e. lamivudine [3TC]/abacavir [ABC]), TAF/FTC should be evaluated on the assumption that TDF/FTC is a different prodrug of TFV, with the same active compound involved (even though with much lower concentrations in plasma and 2.5 times higher in cells). While some data may be still conflicting, recent reviews on different initial ARV regimens pointed out that TDF/TFC may have higher efficacy than $3 T C / A B C{ }^{32}$. Furthermore, the on-going signal of an increased cardiovascular risk in patients taking an $\mathrm{ABC}$-containing regimen beyond and in addition to the well know D: A:D: analyses ${ }^{33}$ makes the FTC/TAF option the safest as far as the NRTIs are concerned.

To date, according to current available data, TAF should be part of CART starting regimens in all naïve patients, with specific consideration to the people over the age of 50 .

\section{cART optimization with TAF-based regimens in experienced patients}

Inexperienced patients, efficacy, and safety of TAF compared to TDF have been established ${ }^{34,35}$. The first randomized, double-blind, controlled study that explored the efficacy and safety of switching to a TAF/ FTC based backbone in combination with a third agent (boosted protease inhibitor, integrase inhibitor, or NNRTI), from TDF/FTC in combination with a third agent, showed noninferiority at 48 weeks, with $94 \%$ of patients in the TAF group being virologically suppressed compared with 93\% in the TDF group (difference $1.3 \%, 95 \% \mathrm{Cl}-2.5-5.1)^{34}$. Moreover, in the same study, with 333 treated in the TAF group and 330 in the TDF group, a low drug discontinuation rate due to adverse events (2.1\% in TAF group compared to $0.9 \%$ in the TDF group) and no cases of proximal renal tubulopathy in either group proved the durability of FTC/ TAF as a backbone and its suitability for future switching strategies regardless the chosen third agent ${ }^{34}$.

The fixed-dose combination of E/C/FTC/TAF is indicated for simplification strategies in virologically suppressed patients (HIV-1 RNA $<50$ copies $/ \mathrm{mL}$ ) for at least 6 months, with no history of treatment failure or resistance to its individual components ${ }^{15,16}$; this switching strategy ensures high rate of efficacy and improvement in proteinuria, albuminuria, and $\mathrm{BMD}^{5}$. This option can be safely applied in patients with mild (eGFR 50$69 \mathrm{~mL} / \mathrm{min}$ ) or moderate (eGFR $30-49 \mathrm{~mL} / \mathrm{min}$ ) renal impairment without dose adjustment ${ }^{5}$. Likewise, studies 1216 and 1160 are evaluating efficacy and safety of a switching strategies from RPV/FTC/TDF (the first) or EFV/FTC/TDF (the latter) to RPV/FTC/TAF in adults virologically suppressed (HIV-1 RNA $<50$ copies per $\mathrm{mL}$ ) for at least 6 months and with creatinine clearance > $50 \mathrm{~mL} / \mathrm{min}$. At week 48, preliminary results showed the switching to RPV/FTC/TAF was well tolerated, with a high rates of virological suppression (> 94\%) and with a statistically significant $(p<0.001)$ decrement of total and tubular proteinuria as well as an increment in BMD at hip and spine ${ }^{35}$.

TAF does not exhibit OAT-dependent cytotoxicity, and it does not accumulate in renal proximal tubules in an OAT-dependent manner, thus justifying the potential benefit on renal function compared to TDF ${ }^{36}$. Moreover, a meta-analysis by Wang et al., including data from six different trials, highlighted the lower decrease in eGFR $\mathrm{R}_{C G}$ and a lower increase of serum creatinine in the TAF group compared to TDF ${ }^{4}$. Renal tu- 
bular proteinuria (retinol binding protein/creatinine and b-2-microglobulin/creatinine) was markedly increased in the TDF group, while this trend was reverted in the TAF group ${ }^{4}$. An on-going trial, registered as NCT02881320, is currently evaluating safety and efficacy in virologically suppressed children and adolescent of Bictegravir/FTC/TAF ${ }^{37}$.

\section{Why TAF-based regimen in experienced patients?}

Patients with stable HIV-RNA suppression, chronic kidney disease (CKD), comorbidity, with an high medication-related burden are all suitable to switch from a TDF based regimen with INSTI or boosted protease inhibitors to a TAF based STR, to ensure long-term benefit on renal function and urinary markers of proximal tubulopathy as well as a significant improvement of $\mathrm{BMD}^{38,39}$.

Even in adult patients with mild to moderately impaired renal function (glomerular filtration rate, GFR, between 30 and $69 \mathrm{~mL} / \mathrm{min}$ ), E/c/FTC/TAF regimen has shown stable and durable improvement of proteinuria and albuminuria ${ }^{38}$.

Due to provide safety and efficacy, TAF should be promptly considered for efficient switching strategies in all experienced patients currently on treatment with FTC/TDF FDC and E/C/FTC/TDF. An on-going study, named NCT02469246, aims to evaluate the efficacy, safety, and tolerability of switching $A B C / 3 T C$ to FTC/ TAF versus maintaining $A B C / 3 T C$ in virologically suppressed adults and may expand indications for future switching strategy to $\mathrm{TAF}^{40}$.

Altogether, these data suggest that switching from TDF to TAF in treatment-experienced patients should minimize the risks of renal impairment and bone mass loss that affect regimens based on TDF; they also suggest that switching from TDF to TAF, not only minimizes these risks but also improves renal tubular function and bone mass density in patients with current, drug-related, unapparent renal and bone damage.

Looking in perspective, the availability of TAF for many simple (often STR) regimens could prompt physicians to switch back to triple standard regimens patients who, because of renal or bone toxicity, were switched in the past to dual regimens; however, this option should be explored in clinical trials before it can be routinely implemented in clinical practice.

Inexperienced patients, TAF should be preferred if the eGFR is decreased more than $>5 \mathrm{~mL} / \mathrm{min}$ and/or the eGFR is $<60 \mathrm{~mL} / \mathrm{min}$. Likewise, osteoporotic pa- tients and postmenopausal woman should benefit of TAF based regimen.

\section{TAF-based regimen in heavily treatment- experienced patients}

Even in heavily treatment-experienced adults, with a high pills burden, a E/c/FTC/TAF plus darunavir 800 mg can reduce the daily intake of pills, improving quality of life and renal function as well as maintaining efficacy and durability ${ }^{41}$. In a phase 3 , open-label, randomized multicenter study, participants were enrolled if were two virological failure and confirmed resistance by historical genotype to at least two different classes of antiretrovirals, no history of INSTI resistance or be INSTI naïve, or be currently suppressed following INSTIs. The primary endpoint was the percentage of participants with HIV-RNA $<50$ copies/mL at week 24 using the US-FDA snapshot algorithm.

Treatment with E/C/FTC/TAF plus darunavir resulted in high rates of virologic suppression $(94 \%$ in new regimen arm vs. $76 \%$ in maintaining CART arm with HIV-RNA < 50 copies $/ \mathrm{mL}$; difference 3.5\%; 95\% $\mathrm{Cl}-3.5 \%$ to $33 \%, p=004)$. Most adverse events were mild or moderate in severity, and no participants discontinued study drug because of an adverse event. No participants had any serious renal adverse event nor discontinued study drugs because of a renal event.

\section{TAF-based regimen use and metabolic profile}

The advents of TAF imply the need to study its metabolic impact, which will need to be considered in PLHIV with diverse baseline cardiovascular risk profile.

The metabolic parameters reported in five RCTs ${ }^{12,23-27,34-42}$ during 48 weeks of therapy showed increased fasting lipid from baseline in the TAF group compared with the TDF group. There were significant differences between the TAF group and the TDF group in the mean changes in total cholesterol (33 vs. $12 \mathrm{mg} / \mathrm{dL}, \mathrm{p}=0.014$ ) and $\mathrm{HDL}$ (7 vs. $3.3 \mathrm{mg} / \mathrm{dL}$, $p=0.008)$, whereas no statistically significant differences between groups were observed in LDL (20 vs. $4.5 \mathrm{mg} / \mathrm{dL}, p=0.124$ ), triglycerides (24 vs. $1.5 \mathrm{mg} / \mathrm{dL}$, $p=0.111$ ), and total cholesterol/HDL ratio (0.1 vs. 0.0 , $p=0.435$ ) in the naive patients. Moreover, for experienced patients, there were significant differences between the TAF group and the TDF group in the mean changes in total cholesterol (17 vs. $1.5 \mathrm{mg} / \mathrm{dL}, \mathrm{p}=0.036$ ) and triglyceride (10.5 vs. $-2 \mathrm{mg} / \mathrm{dL}, \mathrm{p}=0.002$ ), where- 
as no significant differences between groups were observed in LDL (11 vs. $1 \mathrm{mg} / \mathrm{dL}, \mathrm{p}=0.109$ ), HDL (2 vs. $0 \mathrm{mg} / \mathrm{dL}, p=0.184)$, and total cholesterol/HDL ratio (0.15 vs. $0.0, p=0.095)$. These results underline that TAF is lipid-neutral and do not share the "statin-like" phenomenon which characterized TDF.

Nevertheless, CVD prevention cannot be limited to antiretroviral switching versus metabolic friendly drugs but rather introducing statin as a key strategy for cardiovascular risk reduction.

During the aforementioned studies, $5.7 \%$ of all participants in the TAF group and $4.0 \%$ of all participants in the TDF group started lipid-lowering drugs, and no statistically significant difference was found between the groups.

\section{TAF-based regimen use to help BMD}

TAF, regardless BMD reduction at baseline, should be preferred in naive patients as first-regimen to slow diminution in BMD during the following years of treatment. Likewise, due to the reversible nature of the loss bone mass, TAF should be used for switching strategies, disregarding previous CART regimens, both in osteopenia/osteoporotic patients and patients with a consistent BMD reduction greater then physiologically expected. Moreover, adolescents with HIV infection that has not reached the BMD peak yet, may remarkably benefit of TAF based regimen.

\section{TAF-based regimen use to preserve renal function}

In the light of the data showed above, the switch to TAF is, nowadays, mandatory in patients with overt renal impairment, documented by eGFR between 30 and $60 \mathrm{~mL} / \mathrm{min}$ (i.e., CKD Stage III-V), albuminuria, or signs of proximal renal tubular impairment (low molecular weight proteinuria, hypophosphatemia/hyperphosphaturia, normoglycemic glycosuria, and hypouricemia/hyperuricuria), or in patients with a rapid decline of eGFR, although within the normal ranges. However, regardless the renal function, this switch should be also considered in patients with normal renal function but presenting risk factors for kidney diseases such as diabetes, high blood pressure, coinfection with hepatitis viruses, and ART with atazanavir or lopinavir. Moreover, elderly patients should be considered for a switch to TAF. A switch performed before the onset of an eGRF decline in patients at risk could be of strategic importance, considering that in a certain percent-
Table 2. Proposed Agenda for TAF based of patients

When should you definitely use TAF over TDF?

- Patient with low BMD, osteoporosis on DEXA a high risk of major fracture as determined by FRAX.

- Patient with eGFR between 30 and $90 \mathrm{~mL} / \mathrm{min}$ or evidence for proximal tubular dysfunction (e.g., proteinuria) or renal toxicity or other intolerance secondary to TDF

- Older adults

- When the switch is cost-neutral

When should you definitely not use TAF?

- Patient on rifamycin, phenobarbital, carbamazepine

- Pregnant women

- Pre-exposure prophylaxis

- Patients with CKD Stage 4 or 5

- Patients with proven or suspected resistance to the component drugs in any TAF-containing FDC or STR age of patients the TDF-related renal toxicity is incompletely revertible ${ }^{43}$.

The benefits of a shift in preventing renal disease in patients with normal kidney function are less clear and, at present, we have no data to support a possible substitution of TDF with TAF in this case.

\section{Conclusions}

With the advent of effective cART, steady viral suppression has become a common event ${ }^{44}$, and the lifespan of PLWHIV has expanded 45,46 . The spectrum of diseases experienced by PLWHIV has changed, too. AIDS-defining events have become rare 45,47 , while aging with HIV has been linked to non-infectious comorbidities, including cardiovascular disease and hypertension, diabetes mellitus, CKD, osteopenia/osteoporosis, and non-AIDS cancers ${ }^{45,46,48}$.

Chronic HIV infection appears to exacerbate those pathophysiologic processes associated with aging and to increase vulnerability in PLWHIV compared to demographically similar uninfected individuals ${ }^{49}$.

Despite the improvement of long-term tolerability of currently used antiretroviral drugs, long-lasting CART along with aging presumably act as independent risk factors in determining these heterogeneous comorbidities that tend to aggregate into complex multimorbidity patterns ${ }^{50}$. TAF is a relevant adjunct to the current antiretroviral armamentarium as minimizes the long-term effects of CART both on BMD and on renal function that in the past years acted as potential limiting factors of long-lasting CART. Current evidence indicates that switching TDF to TAF is not just a clini- 
Table 3. Proposed Agenda for TAF based on CART regimens

Which patients already treated should change immediately?

Current cART-based

regimen or population

E/C/FTC/TDF

Future cART-based regimen

E/C/FTC/TAF

Boosted PI plus TDF/FTC

boosted PI plus FTC/TAF

Patients coinfected HBV

treated with TDF or

TAF based regimens

entecavir

Rilpivirine/FTC/TDF

Rilpivirine/FTC/TAF

Efavirenz/FTC/TDF

Rilpivirine/FTC/TAF

Darunavir/c plus FTC/TDF

Darunavir/c/FTC/TAF

Which patients already treated might expect to change?

cART-based regimen with virologic success

Raltegravir plus TDF/FTC

Dolutegravir plus TDF/FTC

Boosted PI plus 3TC or FTC

Dolutegravir plus 3TC

cally sound option but is an ethically correct approach, too table 3 .

The TAF cascade should be different for low-income countries (LIC). In fact, a generic formulation of TDF should become available by early $2018,{ }^{51,52}$ while FTC/ TDF in 2021. The price of generic TDF should be relevant in delaying the introduction of TAF in these countries. However, it is necessary to avoid the same mistake made in the past years with other antiretrovirals (i.e., stavudine) maintained with clear evidence of side effects. This Agenda could also aid to prioritize TAF use in patients living in $\mathrm{LIC}$.

\section{Conflicts of interest}

Dr. De Andrea Luca reports grants from ViiV Healthcare, grants from Gilead, personal fees from ViiV Helathcare, personal fees from Gilead, personal fees from Merck Sharp and Dohme, personal fees from Janssen Cilag, outside the submitted work; Dr. Nicola Gianotti reports grants and personal fees from Gilead Science, during the conduct of the study; Dr. Stefano Rusconi grants and personal fees from BMS, grants, personal fees and non-financial support from ViiV, personal fees from MSD, grants, personal fees and non-financial support from Gilead, grants, personal fees and non-financial support from Janssen, outside the submitted work, Dr. Antonio Di Biagio. Dr. Franco Maggiolo, Dr. Giovanni Guaraldi, Dr. Niccoló Riccardi, Dr. Paolo Maggi, Dr. Renato Maserati, Sergio Lo Caputo and Dr. Marco Borderi have nothing to disclose.

\section{References}

1. Guidelines for the Use of Antiretroviral Agents in HIV-1-Infected Adults and Adolescents. Available from: http://www.aidsinfo.nih.gov/guidelines. [Last accessed on 201701 19].

2. Antinori A, Di Biagio A, Marcotullio S, et al. Italian guidelines for the use of antiretroviral agents and the diagnostic-clinical management of HIV-1 infected persons. Update 2016. New Microbiol. 2017;40:86-98.

3. Available from: http://www.gilead.com/news/press-releases/2016/4/us-foodand-drug-administration-approves-descovy-emtricitabine-tenofovir-alafenamide-gileads-third-tafbased-hiv-therapy. [Last accessed on 2018 Feb 27].

4. Wang $H$, Lu X, Yang $X, X u N$. The efficacy and safety of tenofovir alafenamide versus tenofovir disoproxil fumarate in antiretroviral regimens for HIV-1 therapy: meta-analysis. Medicine (Baltimore). 2016;95:e5146.

5. Pozniak A, Arribas JR, Gathe J, et al. Switching to tenofovir alafenamide, coformulated with elvitegravir, cobicistat, and emtricitabine, in HIV-infected patients with renal impairment: 48-week results from a single-arm, multicenter, open-label phase 3 study. J Acquir Immune Defic Syndr. 2016;71:530-7.

6. Lee WA, He GX, Eisenberg E, et al. Selective intracellular activation of a novel prodrug of the human immunodeficiency virus reverse transcriptase inhibitor tenofovir leads to preferential distribution and accumulation in lymphatic tissue. Antimicrob Agents Chemother. 2005;49:1898-906.

7. Birkus G, Kutty N, He GX, et al. Activation of 9-[(R)-2-[[(S)-[[(S)-1- (Isopropoxycarbonyl) ethyl]amino]phenoxyphosphinyl]-methoxy] pro- pyl] adenine (GS-7340) and other tenofovir phosphonoamidate prodrugs by human proteases. Mol Pharmacol. 2008;74:92-100.

8. Ray AS, Fordyce MW, Hitchcock MJ. Tenofovir alafenamide: a novel prodrug of tenofovir for the treatment of human immunodeficiency virus. Antiviral Res. 2016;125:63-70.

9. UNAIDS. World AIDS Day 2014 Report-fact Sheet; 2014. Available from: http://www.unaids.org/en/resources/campaigns/World-AIDS-Day-Report2014/ factsheet. Last accessed on 2018 Feb 27].

10. TenoRes Study Group. Global epidemiology of drug resistance after failure of WHO recommended first-line regimens for adult HIV- Linfection: a multicentre retrospective cohort study. Lancet Infect Dis. 2016;16:565-75

11. Callebaut C, Stepan G, Tian Y, Miller MD. In vitro virology profile of tenofovir alafenamide, a novel oral prodrug of tenofovir with improved antiviral activity compared to that of tenofovir disoproxil fumarate. Antimicrob Agents Chemother. 2015;59:5909-16.

12. Sax PE, Zolopa A, Brar I, et al. Tenofovir alafenamide vs. Tenofovir disoproxil fumarate in single tablet regimens for initial HIV-1 therapy: a randomized phase 2 study. J Acquir Immune Defic Syndr. 2014;67:52-8.

13. Margot NA, Liu Y, Miller MD, Callebaut C. High resistance barrier to tenofovir alafenamide is driven by higher loading of tenofovir diphosphate into target cells compared to tenofovir disoproxil fumarate. Antiviral Res. 2016;132:50-8.

14. EACS Guidelines (8.1). Available from: http://www.eacs.eu. [Last accessed on 2018 Feb 27].

15. Available from: https://www.accessdata.fda.gov/drugsatfda_docs/ nda/2015/207561Orig1s000Approv.pdf. [Last accessed on 2018 Feb 27].

16. Available from: http://www.ema.europa.eu/docs/en_GB/document_library/EPAR_-_Summary_for_the_public/human/004042/WC500197864. pdf. [Last accessed on 2018 Feb 27].

17. Available from: http://www.gilead.com/news/press-releases/2016/3/usfood-and-drug-administration-approves-gileads-second-tafbased-single-tablet-regimen-odefsey-emtricitabine-rilpivirine-tenofovir-alafenamide-for-the-treatment-of-hiv1-infection. [Last accessed on 2018 Feb 27]

18. Available from: http://www.ema.europa.eu/ema/index.jsp?curl=pages/ medicines/human/medicines/004169/human med 002061. jsp\&mid=WCOb01ac058001d124. [Last accessed on 2018 Féb 27]. 
19. Available from: http://www.ema.europa.eu/docs/en GB/document library/EPAR___Summary_for_the_public/human/004094/WC500207653. pdf. [Last accessed on 2018 Feb 27].

20. Available from: http://www.investor.jnj.com/releasedetail. cfm?releaseid=988648 [Last accessed on 2018 Feb 27].

21. Gibson AK, Shah BM, Nambiar PH, Schafer JJ. Tenofovir alafenamide. Ann Pharmacother. 2016:50:942-52.

22. De Clercq E. Tenofovir alafenamide (TAF) as the successor of tenofovir disoproxil fumarate (TDF). Biochem Pharmacol. 2016;119:1-7.

23. Sax PE, Wohl D, Yin MT, et al. Tenofovir alafenamide versus tenofovir disoproxil fumarate, coformulated with elvitegravir, cobicistat, and emtricitabine, for initial treatment of HIV-1 infection: two randomised, double-blind, phase 3, non-inferiority trials. Lancet. 2015;385:2606-15.

24. Arribas JR, Thompson M, Sax PE, et al. Brief report: randomized, doubleblind comparison of tenofovir alafenamide (TAF) vs tenofovir disoproxi fumarate (TDF), each coformulated with elvitegravir, cobicistat, and emtricitabine (E/C/F) for initial HIV-1 treatment: week 144 results. J Acquir Immune Defic Syndr. 2017;75:211-8.

25. Available from: https://www.clinicaltrials.gov/ct2/show/NCT02431247. [Last accessed on 2018 Feb 27].

26. Gaur AH, Kizito H, Prasitsueubsai W, et al. Safety, efficacy, and pharmacokinetics of a single-tablet regimen containing elvitegravir, cobicistat, emtricitabine, and tenofovir alafenamide in treatment-naive, HIVinfected adolescents: a single-arm, open-label trial. Lancet HIV. 2016:3:e561-e568.

27. Mills A, Crofoot G Jr., McDonald C, et al. Tenofovir alafenamide versus tenofovir disoproxil fumarate in the first protease inhibitor-based singletablet regimen for initial HIV-1 therapy: a Randomized phase 2 study. J Acquir Immune Defic Syndr. 2015;69:439-45.

28. Margot N, Cox S, Das M, et al. Infrequent development of drug resistance in HIV-1-infected treatment-naive subjects after 96 weeks of treatment with elvitegravir/cobicistat/emtricitabine/tenofovir alafenamide or elvitegravir/cobicistat/emtricitabine/tenofovir disoproxil fumarate. Antivir Ther. 2017;22:443-6.

29. Imaz A, Podzamczer D. Tenofovir alafenamide, emtricitabine, elvitegravir and cobicistat combination therapy for the treatment of HIV. Expert Rev Anti Infect Ther. 2017;15:195-209.

30. Wohl D, Oka S, Clumeck N, et al. Brief report: a Randomized, doubleblind comparison of tenofovir alafenamide versus tenofovir disoproxil fumarate, each coformulated with elvitegravir, cobicistat, and emtricitabine for initial HIV-1 treatment: Week 96 results. J Acquir Immune Defic Syndr. 2016;72:58-64.

31. Available from: https://www.clinicaltrials.gov/ct2/show/NCT02607956. [Last accessed on 2018 Feb 27].

32. Lee FJ, Amin J, Carr A. Efficacy of initial antiretroviral therapy for HIV-1 infection in adults: a systematic review and meta-analysis of 114 studies with up to 144 weeks' follow-up. PLoS One. 2014;9:e97482.

33. Marcus JL, Neugebauer RS, Leyden WA, et al. Use of abacavir and risk of cardiovascular disease among HIV-infected individuals. J Acquir Immune Defic Syndr. 2016;71:413-9.

34. Gallant JE, Daar ES, Raffi F, et al. Efficacy and safety of tenofovir alafenamide versus tenofovir disoproxil fumarate given as fixed-dose combinations containing emtricitabine as backbones for treatment of HIV-1 infection in virologically suppressed adults: a randomised, double-blind, active-controlled phase 3 trial. Lancet HIV. 2016;3:e158-65.

35. Orkin C DeJesus E, Ramgopal M, et al. Switching from tenofovir disiproxil fumarate to tenofovir alafenamide coformulated with rilpivirine and emtricitabine in virally suppressed adults with HIV-1 infection: a random- ized, double blind, multicentre, phase 3b, non-inferiority study. Lancet HIV. 2017;4:e195-204.

36. Bam RA, Yant SR, Cihlar T. Tenofovir alafenamide is not a substrate for renal organic anion transporters (OATs) and does not exhibit OAT-dependent cytotoxicity. Antivir Ther. 2014;19:687-92.

37. Available from: https://www.clinicaltrials.gov/ct2/show/study/NCT0288 1320?view=results. [Last accessed on 2018 Feb 27].

38. Post FA, Tebas $P$, Clarke A, et al. Brief report: switching to tenofovir alafenamide, coformulated with elvitegravir, cobicistat, and emtricitabine, in HIV-infected adults with renal impairment: 96-week results from a single-arm, multicenter, open-label phase 3 study. J Acquir Immune Defic Syndr. 2017;74:180-4.

39. Bonora S, Calcagno A, Trentalange A, Di Perri G. Elvitegravir, cobicistat, emtricitabine and tenofovir alafenamide for the treatment of HIV in adults. Expert Opin Pharmacother 2016;17:409-19.

40. Switch Study to Evaluate F/TAF in HIV-1 Infected Adults Who Are Virologically Suppressed on Regimens Containing ABC/3TC. Available from: https://www.clinicaltrials.gov/ct2/show/NCT02469246. [Last accessed on 2017 May 22]

41. Huhn GD, Tebas P Gallant J, et al. A randomized, open-label trial to evaluate switching to elvitegravir/Cobicistat/Emtricitabine/Tenofovir alafenamide plus darunavir in treatment-experienced HIV-1-infected adults. J Acquir Immune Defic Syndr. 2017:74:193-200.

42. Mills A, Arribas JR, Andrade-Villanueva J, et al. Switching from tenofovir disoproxil fumarate to tenofovir alafenamide in antiretroviral regimens for virologically suppressed adults with HIV-1 infection: a randomised, active-controlled, multicentre, open-label, phase 3, non-inferiority study. Lancet Infect Dis. 2016;16:43-52.

43. Brennan A, Evans D, Maskew M, Naicker S, Ive P, Sanne I, Maotoe T, Fox M. Relationship between renal dysfunction, nephrotoxicity and death among HIV adults on tenofovir. AIDS. 2011 Aug 24;25(13):1603-9

44. McKinnell JA, Willig JH. Westfall AO, et al. Antiretroviral prescribing patterns in treatment-naïve patients in the united states. AIDS Patient Care STDS. 2010;24:79-85.

45. Guaraldi G, Orlando G, Zona S, et al. Premature age-related comorbidities among HIV-infected persons compared with the general population. Clin Infect Dis. 2011;53:1120-6.

46. Losina E, Schackman BR, Sadownik SN, et al. Racial and sex disparities in life expectancy losses among HIV-infected persons in the United States: impact of risk behavior, late initiation, and early discontinuation of antiretroviral therapy. Clin Infect Dis. 2009;49:1570-8.

47. d'Arminio Monforte A, Sabin CA, et al. The changing incidence of AIDS events in patients receiving highly active antiretroviral therapy. Arch Intern Med. 2005;165:416-23.

48. Schouten J, Wit FW, Stolte IG, et al. Cross-sectional comparison of the prevalence of age-associated comorbidities and their risk factors between HIV-infected and uninfected individuals: the AGEhIV cohort study. Clin Infect Dis. 2014;59:1787-97.

49. Justice AC. HIV and aging: time for a new paradigm. Curr HIV/AIDS Rep. 2010;7:69-76

50. Deeks SG. Immune dysfunction, inflammation, and accelerated aging in patients on antiretroviral therapy. Top HIV Med. 2009;17:118-23.

51. Phillips AN, Neaton J, Lundgren JD. The role of HIV in serious diseases other than AIDS. AIDS. 2008;22:2409-18.

52. Hill AM, Pozniak AL. How can we achieve universal access to low-cost treatment for HIV? J Virus Erad. 2016;2:193-7. 